\title{
ERRATA
}

\section{Erratum: "Charge transformer to enhance noise performance of single-electron transistor amplifiers in high-capacitance applications" [Appl. Phys. Lett. 80, 142 (2002)]}

K. M. Lewis and Ç. Kurdak ${ }^{\text {a) }}$

Physics Department, University of Michigan, Ann Arbor, Michigan 48109

S. Krishna ${ }^{\text {b) }}$ and P. Bhattacharya

Department of Electrical Engineering and Computer Science, University of Michigan, Ann Arbor, Michigan 48109

[DOI: $10.1063 / 1.1452815]$

Due to a production error, Fig. 3 was reproduced in the printed journal in grayscale, rather than in color. AIP apologizes for this error. The color version of Fig. 3 is available in the online versions of the article.

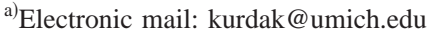

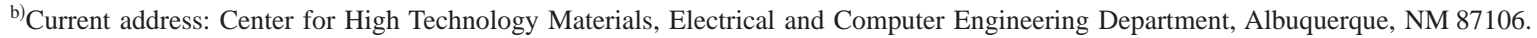

